\title{
Understanding deep-water striation patterns and predicting the waveguide invariant as a distribution depending on range and depth
}

Rémi Emmetière, Julien Bonnel, Marie Géhant, Xavier Cristol, and Thierry Chonavel

Citation: The Journal of the Acoustical Society of America 143, 3444 (2018); doi: 10.1121/1.5040982

View online: https://doi.org/10.1121/1.5040982

View Table of Contents: http://asa.scitation.org/toc/jas/143/6

Published by the Acoustical Society of America

\section{Articles you may be interested in}

Stochastic matched-field localization of an acoustic source based on principles of Riemannian geometry

The Journal of the Acoustical Society of America 143, 3628 (2018); 10.1121/1.5040492

Modeling underwater noise propagation from marine hydrokinetic power devices through a time-domain, velocity-pressure solution

The Journal of the Acoustical Society of America 143, 3242 (2018); 10.1121/1.5039839

Acoustic scattering from a cylindrical shell with an internal rigid plate: Analysis and experiment

The Journal of the Acoustical Society of America 143, 3332 (2018); 10.1121/1.5040469

Source localization using deep neural networks in a shallow water environment

The Journal of the Acoustical Society of America 143, 2922 (2018); 10.1121/1.5036725

Striation-based source depth estimation with a vertical line array in the deep ocean

The Journal of the Acoustical Society of America 143, EL8 (2018); 10.1121/1.5020267

Passive, broadband suppression of radiation of low-frequency sound

The Journal of the Acoustical Society of America 143, EL67 (2018); 10.1121/1.5022192 


\title{
Understanding deep-water striation patterns and predicting the waveguide invariant as a distribution depending on range and depth
}

\author{
Rémi Emmetière ${ }^{a)}$ \\ Ecole Nationale Supérieure de Techniques Avancées Bretagne, Lab-STICC (UMR CNRS 6285), \\ 2 rue François Verny, 29806 Brest Cedex 9, France \\ Julien Bonnel \\ Woods Hole Oceanographic Institution, 266 Woods Hole Road, Woods Hole, Massachusetts 02543-1050, USA \\ Marie Géhant and Xavier Cristol \\ Thales Underwater System, 525 Route des Dolines, 06560 Sophia-Antipolis Cedex, France \\ Thierry Chonavel \\ Institut Mines Télécom Atlantique, Lab-STICC, Université Bretagne Loire, F-29238 Brest, France
}

(Received 1 December 2017; revised 14 April 2018; accepted 21 May 2018; published online 8 June 2018)

The Waveguide Invariant (WI) theory has been introduced to quantify the orientation of the intensity interference patterns in a range-frequency domain. When the sound speed is constant over the water column, the WI is a scalar with the canonical value of 1 . But, when considering shallow waters with a stratified sound speed profile, the WI ceases to be constant and is more appropriately described by a distribution, which is mainly sensitive to source/receiver depths. Such configurations have been widely investigated, with practical applications including passive source localization. However, in deep waters, the interference pattern is much more complex and variable. In fact the observed WI varies with source/receiver depth but it also varies very quickly with source-array range. In this paper, the authors investigate two phenomena responsible for this variability, namely the dominance of the acoustic field by groups of modes and the frequency dependence of the eigenmodes. Using a ray-mode approach, these two features are integrated in a WI distribution derivation. Their importance in deep-water is validated by testing the calculated WI distribution against a reference distribution directly measured on synthetic data. The proposed WI derivation provides a thorough way to predict and understand the striation patterns in deep-water context.

(C) 2018 Acoustical Society of America. https://doi.org/10.1121/1.5040982

[BTH]

Pages: $3444-3454$

\section{INTRODUCTION}

An underwater waveguide is bounded by the surface and the seabed, leading to multi-path/multi-mode propagation so that the acoustic field exhibits interferences. When looking at the acoustic intensity of a broadband signal over horizontal (range) aperture, these interferences build a structured pattern that can be used for source localization or the waveguide properties inversion. At low frequencies, interferences are stable enough to be observable ${ }^{1}$ and take the form of striations. The sum of all the striations is often called interference pattern. First highlighted by Weston and Stevens on a moving noisy ship, ${ }^{2}$ Chuprov formalized the problem using the Waveguide Invariant (WI) theory ${ }^{3}$ which describes the slope of those striations. More recent investigators developed and detailed this theory adopting a normal mode point of view, 4,5 a geometric ray point of view, ${ }^{6}$ and both points of view. ${ }^{7,8}$ Most of these studies consider range independent shallow water waveguides where the propagation is dominated by interface reflections at the surface and seabed so that effects of a sound-speed profile (SSP) are generally neglected. It has

${ }^{\text {a)} E l e c t r o n i c ~ m a i l: ~ r e m i . e m m e t i e r e @ e n s t a-b r e t a g n e . o r g ~}$ been shown that, in this case, all striations have the same slope and, as a result, the WI is a scalar traditionally denoted by $\beta$. In most shallow water environments, $\beta$ is roughly constant with a value close to 1 . The apparent simplicity of the WI gave rise to an exhaustive set of applications (passive localization, ${ }^{9,10}$ geo-acoustics inversion, ${ }^{11}$ active sonar, ${ }^{12,13}$ source separation, ${ }^{14}$ dispersion compensation ${ }^{15,16}$ ).

On the other hand, Baggeroer ${ }^{17}$ and Rouseff and Spindel ${ }^{18}$ simultaneously reminded that considering the WI as a constant is not realistic for stratified SSPs, where refraction becomes non-negligible. For these environments, the striation pattern is more accurately described by a WI distribution. This distribution can be directly measured on data by applying a two-dimensional Fast Fourier Transform (2DFFT) on $I(r, f)$, the range-frequency intensity. This generic process seems to have been proposed independently by Rouseff and Spinde, ${ }^{18}$ Baggereor, ${ }^{17}$ and Yang. ${ }^{19}$ It allows estimation of the WI distribution in any environment of interest (shallow or deep), assuming that the input data has a range/frequency resolution that allows to resolve the striations. The WI distribution obtained using this process will be called the "reference" distribution throughout the paper, and will be noted $E_{\beta}$. Although the reference WI distribution can 
be directly measured on data, it does not bring any physical insight on the phenomena that drive the WI values. Rouseff and Spindel also provided an analytical derivation of the image processing based on the normal mode expansion. ${ }^{18} \mathrm{It}$ shows that the distribution is dependent on source and receiver depths. Making use of this physical understanding, the WI distribution has been recently used to infer source depth in shallow water. ${ }^{20}$ But to achieve their derivation, Rouseff and Spindel used several assumptions, including an infinite range averaging, so that it does not catch the range dependence of the striation pattern that typically occurs in deep-water. As a result, it is appropriate for shallow water but not for deep water configurations.

In deep water environments, the SSPs are highly stratified. As discussed in Chuprov's original paper, ${ }^{3}$ the shape of the SSP in deep-water gives rise to numerous widely spread values of the WI, especially for low order modes. As a result, the striation pattern (and thus the WI) must be described by a wide distribution, whose values depend on the SSP. Unfortunately, Rouseff and Spindel's work ${ }^{18}$ is not valid in deep water, and today no method exists to predict and understand the WI distribution in this context. For this reason, WI has not encountered much success in deep water, and very few studies attempt to use it for signal processing applications. ${ }^{21-23}$

In this paper, we propose a new derivation for the WI distribution in deep water. In particular, we investigate two reasons that contribute to the variability of the WI for a fixed SSP. On one hand, the existence of convergence and shadow zones, resulting from the dominance of the acoustic field by groups of modes, involves dramatical changes in the interference pattern depending on source-array range and depths. ${ }^{24}$ On the other hand, and especially within the first tens of kilometers, the WI itself varies with source-to-array range because of the frequency dependence of the eigenmodes. The main contribution of this work is to take directly into account these two deep water behaviors in the WI calculation. Our derivation thus allows to predict and understand the WI distribution in a deep-water context from the point of view of the normal modes theory. Despite the complexity of the WI in deep water context, a thorough understanding of the striation pattern could lead to the development of new source localization methods that are based on the WI distribution. Today, such methods allow source depth discrimination, but are restricted to shallow water. ${ }^{18}$

Within this framework, we demonstrate why and how the deep water WI distribution is varying with the source-receiver configuration and how to predict locally such a distribution. For this purpose, in Sec. II, we first recall the classical definition of the WI, and the 2D-FFT process that is used to evaluate the reference WI distribution. As a reminder, this reference distribution can be evaluated on simulated/experimental data, both in shallow and deep water. It will be noted $E_{\beta}$ in the following. Then, in Sec. III, we propose a first method to predict and intuitively understand $E_{\beta}$ by taking into account the modal group dominance. To do so, the interference pattern is computed as the sum of interference striations produced by subsets of modes which are in-phase with their neighborhoods. We show that this derivation of $E_{\beta}$ is not enough to explain the sensitivity of the interference pattern with respect to range.
Second, we complete the previous WI calculation by introducing the frequency dependence of the eigenmodes using a raymode approach. It leads to the full prediction of $E_{\beta}$. The WI distribution evaluated according this new technique closely matches the reference striation pattern. Finally, in Sec. IV, we focus on specific configurations to highlight some interesting features of the striation pattern in deep water.

\section{BASICS OF THE WI}

\section{A. Definition of the WI}

According to normal mode theory ${ }^{8}$ in stratified range independent marine environments, the contribution to the total acoustic pressure of mode $m$, for a point source at depth $z_{s}$, a receiver at depth $z_{r}$ and range $r$ may classically be written as follows:

$$
p_{m}\left(z_{s}, z_{r}, r, \omega\right) \propto \frac{\psi_{m}\left(z_{s}, \omega\right) \psi_{m}\left(z_{r}, \omega\right)}{\sqrt{k_{m}(\omega) r}} e^{i k_{m}(\omega) r},
$$

where $\psi_{m}$ is the eigenmode and $k_{m}$ the associated horizontal wavenumber. For convenience, the horizontal wavenumbers are assumed to be real, but the analysis is easily generalized to lossy media. The total pressure is a sum of $N$ propagating modes with amplitudes $A_{m}$ and phases $\phi_{m}$,

$$
\begin{aligned}
p\left(z_{s}, z_{r}, r, \omega\right) & \equiv \sum_{m=1}^{N} p_{m}\left(z_{s}, z_{r}, r, \omega\right) \\
& =\frac{1}{\sqrt{r}} \sum_{m=1}^{N} A_{m}\left(z_{s}, z_{r}, \omega\right) e^{i \phi_{m}\left(z_{s}, z_{r}, r, \omega\right)} .
\end{aligned}
$$

This coherent sum will produce constructive and destructive interferences. Frequency dependence of $A_{m}$ is usually ignored, ${ }^{8}$ but this is an approximation, valid in the ideal case of isovelocity channels with pressure-release or rigid boundaries. Considering that the cylindrical divergence $1 / \sqrt{r}$ varies slowly with respect to $r$ compared to the exponential term, it is also routinely ignored. Denoting $\Delta \phi_{m n}=\phi_{m}-\phi_{n}$ the phase shift between modes, one can expand the acoustic intensity and extract the interference pattern ${ }^{8}$

$$
\begin{aligned}
I\left(z_{s}, z_{r}, r, \omega\right) \equiv & \left|p\left(z_{s}, z_{r}, r, \omega\right)\right|^{2} \\
\propto & \sum_{m, n, m \neq n} A_{m}\left(z_{s}, z_{r}\right) A_{n}\left(z_{s}, z_{r}\right) \\
& \times \cos \left(\Delta \phi_{m n}\left(z_{s}, z_{r}, r, \omega\right)\right) .
\end{aligned}
$$

The total pressure field exhibits structured cosine striations, as illustrated in Fig. 1 for a Pekeris waveguide. For each pair of modes, one can define the following quantity:

$$
I_{m n}\left(z_{s}, z_{r}, r, \omega\right)=A_{m}\left(z_{s}, z_{r}\right) A_{n}\left(z_{s}, z_{r}\right) \cos \left(\Delta \phi_{m n}\left(z_{s}, z_{r}, r, \omega\right)\right),
$$

which represents the part of the interference pattern due to the interference between mode $m$ and $n$. Since frequency dependence of $A_{m}$ is approximately ignored, the slope of the striations is only due to the frequency shift of the cosine term of Eq. (4). In the following, the amplitude $A_{m} A_{n}$ is called the "interference excitation". 


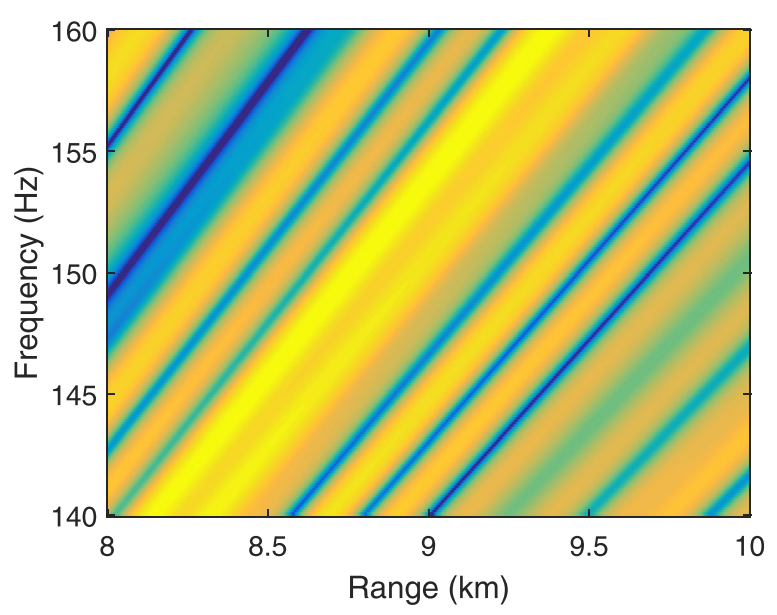

FIG. 1. (Color online) Acoustic intensity of a flat spectrum broadband signal in a $150 \mathrm{~m}$ depth Pekeris waveguide over a $2 \mathrm{~km}$ long HLA. $c_{\text {water }}=1500 \mathrm{~m} / \mathrm{s}, c_{\text {seabed }}=1700 \mathrm{~m} / \mathrm{s}$ and $z_{s}=z_{r}=100 \mathrm{~m}$.

Two different definitions of the WI have been proposed in literature:

(1) The reference WI definition. From the range-frequency variability of the acoustic intensity $I_{m n}$, the WI is properly defined as the slope of the interference striation in log-log space $^{4,8}$ visible through a window $(r, \omega)$ with bandwidth $B$ and range aperture $L$. Introduced this way, it can be unambiguously evaluated by assessing the slope of an iso-intensity line in the log-log representation of the range-frequency intensity function

$$
\beta_{m n}\left(z_{r}, z_{s}, r, \omega\right)=\left.\frac{d(\log \omega)}{d(\log r)}\right|_{I_{m n}=c s t}=\left.\frac{r}{\omega} \frac{d \omega}{d r}\right|_{I_{m n}=c s t} .
$$

Note that Eq. (5) is often defined for the total intensity $I$, because it is usually impossible to extract the contribution $I_{m n}$ of any pair of modes from the total striation pattern $I$.

(2) The definition relying on normal mode expression. The WI resulting from the interference between modes $m$ and $n$ can be rewritten ${ }^{4,8}$ as

$$
\beta_{m n}\left(z_{s}, z_{r}, r, \omega\right)=-\frac{r}{\omega} \frac{\frac{\delta \Delta \phi_{m n}\left(z_{s}, z_{r}, r, \omega\right)}{\delta r}}{\frac{\delta \Delta \phi_{m n}\left(z_{s}, z_{r}, r, \omega\right)}{\delta \omega}} .
$$

If $A_{m}$ depends neither on range nor on frequency, Eq. (6) is an exact formulation. In the literature, it is typically used assuming that $\psi_{m}$ is a real valued function that locally (around $\omega_{0}$ ) does not depend on frequency. In other words, the classical assumption states that

$$
\phi_{m}(r, \omega)=k_{m}(\omega) r
$$

and

$$
A_{m}\left(z_{r}, z_{s}\right)=\left.\frac{\psi_{m}\left(z_{s}, \omega\right) \psi_{m}\left(z_{r}, \omega\right)}{\sqrt{k_{m}(\omega)}}\right|_{\omega=\omega_{0}} .
$$

Inserting Eq. (7) into Eq. (4), the source/receiver depths and range dependence in the WI definition cancels out. ${ }^{4,8}$

$$
\beta_{m n}(\omega)=-\frac{1}{\omega} \frac{\Delta k_{m n}(\omega)}{\frac{\delta \Delta k_{m n}(\omega)}{\delta \omega}}
$$

where $\Delta k_{m n}=k_{m}-k_{n}$. Under the same assumption, the frequency shift of the cosine in Eq. (4) can be physically understood $^{4,8}$ in terms of the horizontal phase slowness $S_{p, m}^{h}(\omega)=k_{m}(\omega) / \omega$ and the horizontal group slowness $S_{g, m}^{h, m}(\omega)=\delta k_{m}(\omega) / \delta \omega$. Then Eq. (9) is often reformulated $^{4,8}$ as follows:

$$
\beta_{m n}(\omega)=-\frac{\Delta S_{p, m n}^{h}(\omega)}{\Delta S_{g, m n}^{h}(\omega)}
$$

where $\Delta S_{p, m n}^{h}=S_{p, m}^{h}(\omega)-S_{p, n}^{h}(\omega)$ and $\Delta S_{g, m n}^{h}=S_{g, m}^{h}(\omega)$ $-S_{g, n}^{h}(\omega)$. It is important to note that Eqs. (9) and (10) are an approximation, because the eigenmode $\psi_{m}$ depends on frequency. Another phase term must be considered if $\psi_{m}$ significantly and quickly changes with frequency, as it is generally the case in deep oceans.

In a deep water context, we will see in Sec. III that Eq. (9) or Eq. (10) is not accurate enough to describe the reference WI in Eq. (5), especially for short ranges. One can already note that there are as many $\beta$-values as pairs of modes $(m, n)$. Depending on which modes are interfering, several striations possibly coexist, building a complex striation pattern which is better quantified by a distribution of $\beta$, noted $E_{\beta}$, as it has been suggested in Ref. 18 in a shallow water context.

\section{B. Evaluate the reference WI distribution}

The goal of this section is to introduce the method and the notations used to assess the reference WI distribution $E_{\beta}$ from a given sampled range-frequency picture $I(r, f)$ like the one plotted in Fig. 2(a). For these simulated data and for all the subsequent simulations, the considered environment is a typical Mediterranean summer channel, and is modeled with the values listed in Table I and illustrated in Fig. 3(a). This kind of environment with a single underwater channel can be modeled with two speed gradients, one above and one below the minimum of celerity. The eigenmodes $\psi_{m}$ along with the associated eigenvalues $k_{m}$ are evaluated using the numerical KRAKEN code. $^{25}$

As defined in Eq. (5), the WI is related to the slope of interference striations. As stated in Sec. I, one way to empirically measure the WI is to use a 2D-FFT. The corresponding method, described in Refs. 4, 18, and 26 will be briefly reviewed below.

The 2D-FFT of a given image $I(r, f)$ with bandwidth $B$ and range aperture $L$ is defined by

$$
\tilde{I}(x, y)=\left|\int_{f_{0}-B / 2}^{f_{0}+B / 2} \int_{r_{0}-L / 2}^{r_{0}+L / 2} I(r, f) e^{-i 2 \pi(x r+y f)} d r d f\right|,
$$

where $r_{0}$ and $f_{0}$ are the mean values of axis $r$ and $f$. Variables $x$ (in $\mathrm{m}^{-1}$ ) and $y$ (in $\mathrm{s}$ ) are Fourier conjugate variables of the range axis and the frequency axis, respectively. 
(a)

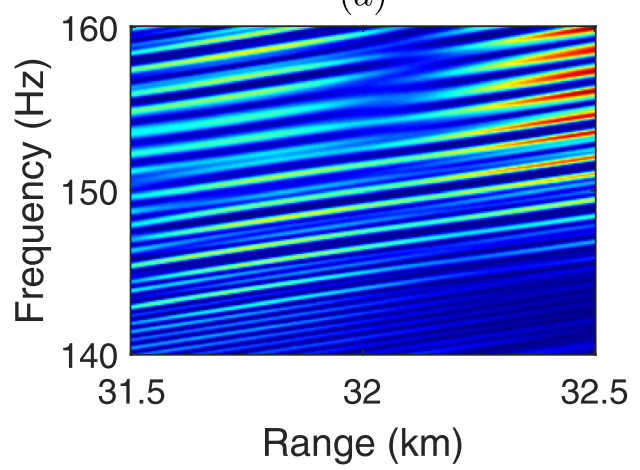

(c)

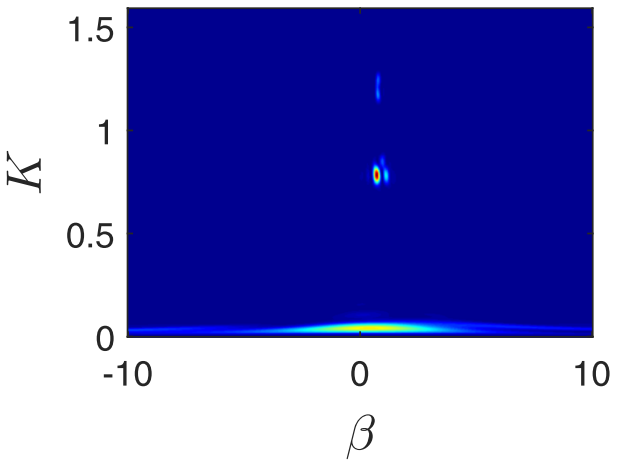

According to Eq. (3), $I(r, f)$ is a sum of a finite number of windowed cosines. Then, the energy in $\tilde{I}(x, y)$ is sparsely distributed as a sum of a finite number of Diracs function convoluted by sinc (cardinal sine) functions. Figure 2(b) gives an example of this sparsity. Up to a constant, Eq. (11) can be approached by

$$
\begin{aligned}
& \sum_{m, n, m \neq n} A_{m} A_{n} \operatorname{sinc}(\pi x L) \operatorname{sinc}(\pi y B) \\
& \quad *\left(\delta\left(x-x_{m n}, y-y_{m n}\right)+\delta\left(x_{m n}-x, y_{m n}-y\right)\right),
\end{aligned}
$$

where $x_{m n}$ and $y_{m n}$ denote the frequencies of the interference striations between modes $m$ and $n$ along, respectively, the range $r$ and the frequency axis $f . \delta$ is the Dirac delta functional and $*$ denotes convolution. One can give a clearer representation of the WI information with another set of variables, ${ }^{4,16}$ closely related to polar coordinate system. By replacing the slope in Eq. (5) by its expression in the Fourier domain one obtains

$$
\beta=-\frac{r x}{f} \frac{x}{y},
$$

and we arbitrarily chose

TABLE I. Typical summer deep water Mediterranean environment.

\begin{tabular}{lccc}
\hline \hline depth $[\mathrm{m}]$ & sound speed $[\mathrm{m} / \mathrm{s}]$ & density $\left[\mathrm{kg} / \mathrm{m}^{3}\right]$ & attenuation $[\mathrm{dB} / \lambda]$ \\
\hline 0 & 1530 & 1030 & 0 \\
100 & 1500 & 1030 & 0 \\
2500 & 1550 & 1030 & 0 \\
2500 & 1700 & 1700 & 0.6 \\
$\infty$ & 1700 & 1700 & 0.6 \\
\hline
\end{tabular}

(b)

FIG. 2. (Color online) All steps of the assessment of $E_{\beta}\left(z_{s}, z_{r}, r\right)$ for the configuration setting $z_{s}=10 \mathrm{~m}, z_{r}=100 \mathrm{~m}$, $r=32 \mathrm{~km}$ and $f=150 \mathrm{~Hz}$. (a) The range-frequency image $I(r, f)$. (b) The 2D-FFT of $I(r, f)$. (c) 2D-FFT in the polar coordinates. (d) The WI distribution $E_{\beta}\left(z_{s}, z_{r}, r\right)$.
An example of such a transform is displayed by Fig. 2(c). Summing up over $K$ gives the reference WI distribution $E_{\beta}$, which is plotted in Fig. 2(d). High energy denotes the presence of striation slope at $\beta .^{4,16}$ This distribution is associated with the specific set of parameters $\left(z_{r}, z_{s}, r, f\right)$ chosen to generate the picture $I(r, f)$. Since this is a direct measurement and it does not make any approximation, $E_{\beta}$ is considered in this paper as our reference distribution, or ground truth. Influences of frequency, bandwidth, and array aperture on $E_{\beta}$ will not be discussed in this paper in order to focus on the depth/range dependence.

By repeating this procedure at several successive depths or ranges, one can clearly observe the effects of source depth on $E_{\beta}$, as shown by Fig. 4(a), or the effects of source range, as demonstrated by Fig. 4(b). The sensitivity of $E_{\beta}$ on source depth has been well discussed ${ }^{16}$ for shallow water configurations. It is attributed to the interference excitation $A_{m} A_{n}$ in Eq. (4), which is a function of source/receiver depths. It has given rise to depth estimation applications. ${ }^{20,27}$ But, it is clear from Fig. 4(b) that $E_{\beta}$ also depends on range. However, neither the interference excitation nor the WI as derived in Eq. (9) or Eq. (10) can explain this range dependence. As noted by Cockrell in his $\mathrm{PhD}$ manuscript (Appendix A2 of Ref. 4), the range dependence of the striation has been observed but it is "a topic that is not well studied".

Section III first shows how to derive $E_{\beta}$ as an indirect function of range by taking into account the dominance in the field of groups of modes. This idea is mentioned in several papers ${ }^{3,4,12}$ but it has never been introduce directly in a derivation of $E_{\beta}$. Second, we derive the WI from Eq. (6) as an explicit function of range and depth. We will show that 

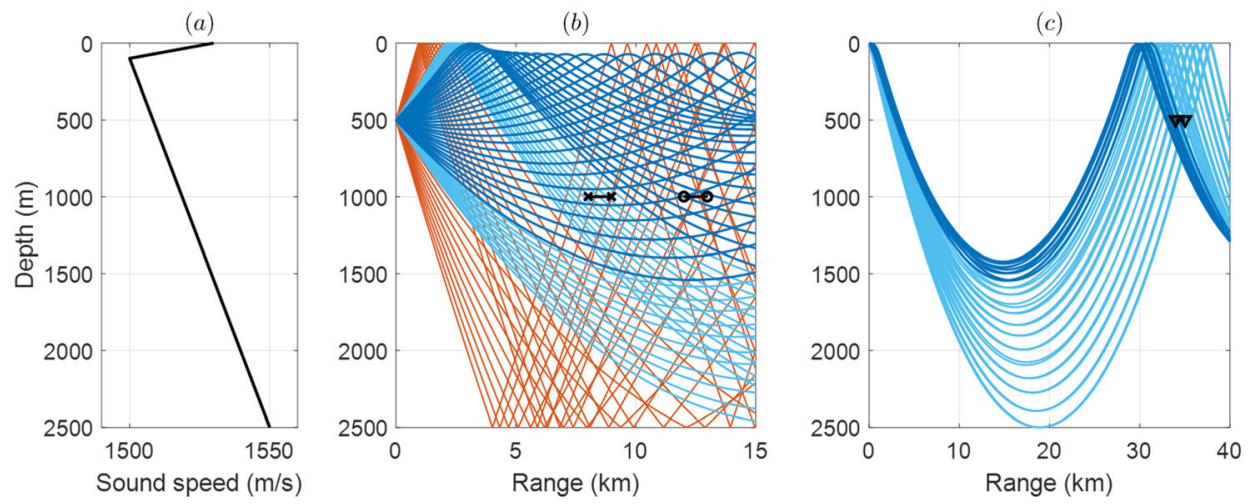

FIG. 3. (Color online) (a) Mediterranean sound speed profile considered in this study, and ray tracing in this environment with (b) $z_{s}=500 \mathrm{~m}$ and (c) $z_{s}=10 \mathrm{~m}$. Three types of rays exist: top/bottom refracted rays (dark blue), top reflected bottom refracted rays (light blue), and top/bottom reflected rays (orange). The position of the HLAs that are used in the three test cases are superimposed on (b) and (c) as black crosses, circles, and triangles. For representation convenience, top/bottom reflected rays are not drawn in (c).

coupling the two approaches allows a thorough formula of the WI distribution.

\section{THE DEEP WATER WI DISTRIBUTION}

\section{A. On the dominance of modal groups}

In practice, in deep water the acoustic fields are dominated by few groups of modes. In fact, even at low frequency (down to $10 \mathrm{~Hz}$ ), the density of modes is high enough, so that groups of neighboring modes merge and behave together like "fuzzy rays." ${ }^{28}$ For a specified source/receiver configuration, visible interferences do not arise from all modes, but occur only between modes which are within those groups. This introduces the concept of "modal dominance"3,29,30 in the calculation of $E_{\beta}$. This feature is mentioned in the WI literature, ${ }^{3,4}$ but as far as we know, no published investigation explicitly integrates this idea into a normal modes derivation of $E_{\beta}$. According to Eq. (2), the complex pressure field is a sum of $N$ modes with local

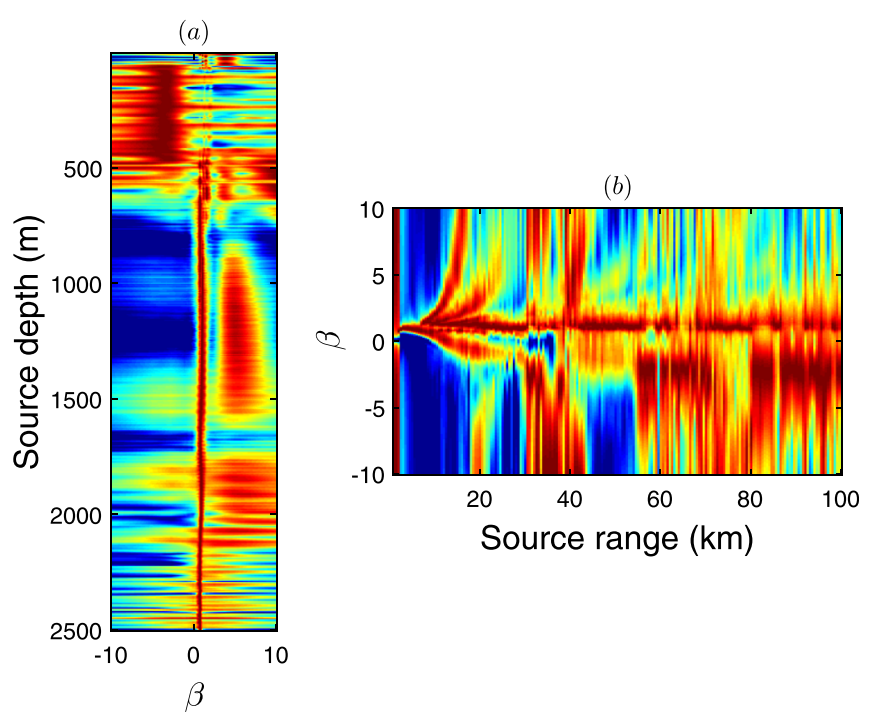

FIG. 4. (Color online) A noise realization with bandwidth $B=20 \mathrm{~Hz}$ centered on $f=150 \mathrm{~Hz}$ is simulated on an HLA with aperture $L=1 \mathrm{~km}$. (a) $E_{\beta}(z)$ with varying source depth. Other parameters are set at $z_{r}=100 \mathrm{~m}$ and $r=50 \mathrm{~km}$. (b) $E_{\beta}(r)$ with varying source range. Other parameters are set at $z_{r}=1000 \mathrm{~m}$ and $z_{s}=500 \mathrm{~m}$. maxima occurring when adjacent modes constructively interfere. Physically, this may occur when two adjacent modes $m$ and $m+1$ are in phase

$$
\Delta \phi_{m, m+1}(r, \omega)=2 p \pi,
$$

with $p$ an integer number. Nevertheless at any given precise point $(r, \omega)$, it is unusual to exactly find integer values of $m$ that strictly verify Eq. (15). In practice, we track the evolution of $p$ as a function of $m$ and by interpolation we find modal interference indexes $i$ (which are often not integers) corresponding to integer values of $p$. The factitious group of neighboring modes centered on mode $m_{p}=i+0.5$ is then referred to as dominant. Note that the additional factor +0.5 arises because the integer value of $p$ found using Eq. (15) represents the interference between neighboring modes $i$ and $i+1$.

The major contribution to the pressure field of a dominant group is given by the adjacent modes $(m, m+1)$ surrounding $m_{p}$. However, the horizontal period of interference striations ${ }^{3,31}$ resulting from the interference of any adjacent modes is given by

$$
D_{m, m+1}(\omega)=\frac{2 \pi}{\Delta k_{m, m+1}(\omega)} .
$$

A key point is that these striations have an interference period that is longer than the length of a realistic horizontal array and so might not be observed in practice. It means that the WI will be calculated only between dominant groups and not within dominant groups. We consider that each dominant group behaves like a unique equivalent mode which is the central mode $m_{p}$. Any modal quantity $\left(k_{m_{p}}, k_{z, m_{p}}, \phi_{m_{p}}, A_{m_{p}}, \ldots\right)$ associated to a non-integer $m_{p}$ can be evaluated through interpolation with known quantities upon modes. Using equivalent modes $m_{p}$ and $m_{q}$ of two dominant groups, the WI is

$$
\beta_{m_{p} m_{q}}(\omega)=-\frac{1}{\omega} \frac{\Delta k_{m_{p} m_{q}}(\omega)}{\frac{\delta \Delta k_{m_{p} m_{q}}(\omega)}{\delta \omega}} .
$$

According to Eq. (17), the WI appears as indirectly range dependent through the modal dominance, since Eq. (15) is 
itself range dependent. As discussed previously, the WI can be defined in terms of phase/group slowness, and

$$
\beta_{m_{p} m_{q}}(\omega)=-\frac{\Delta S_{p, m_{p} m_{q}}^{h}(\omega)}{\Delta S_{g, m_{p} m_{q}}^{h}(\omega)} .
$$

In order to obtain results consistent with the reference $E_{\beta}$, evaluated in Sec. II B, we reconstruct a WI distribution as a sum of equivalent modes interferences (i.e., striations slope at $\beta_{m_{p} m_{q}}$ weighted by its interference amplitude $\left.A_{m_{p}} A_{m_{q}}\right)$. In terms of equivalent modes, Eq. (12) can be rewritten by changing $m$, respectively $n$, with $m_{p}$, respectively $m_{q}$. The horizontal spatial frequency $x_{m_{p} m_{q}}$ of the interference striation resulting from the interference of $m_{p}$ and $m_{q}$ is given by

$$
x_{m_{p} m_{q}}=\frac{\Delta k_{m_{p} m_{q}}}{2 \pi}
$$

The vertical spatial frequency $y_{m_{p} m_{q}}$ can be evaluated using Eqs. (18) and (13)

$$
y_{m_{p} m_{q}}=-\frac{r}{f} \frac{x_{m_{p} m_{q}}}{\beta_{m_{p} m_{q}}} .
$$

In physical terms, $y_{m_{p} m_{q}}$ corresponds to the time delay between the arrival of modes $m_{p}$ and $m_{q}$. Following the same variable changes as in Sec. II B, a WI distribution is calculated. It is referred to as an approximate prediction, noted $\hat{E}_{\beta}$, in contrast with the reference $E_{\beta}$ directly evaluated on $I(r, f)$. For the example of Sec. II B, $E_{\beta}$ is plotted in Fig. 5. If the modal group dominance is enough to explain the range dependence, it should match $E_{\beta}$ displayed in Fig. 4(b).

Unfortunately when comparing the two distributions we observe that the predicted distribution $\hat{E}_{\beta}$ fails to fully reproduce the reference striation pattern, especially at short ranges. This explicitly demonstrates that a coupled model with $\beta$ as defined in Eq. (10) and the modal dominance is not enough to explain striation patterns in deep water. Sections III B-III D supplement the method with an Amplitude Modulation/ Frequency Modulation (AM-FM) expansion of the eigenmode. The objective is to extract its oscillatory part as a phase

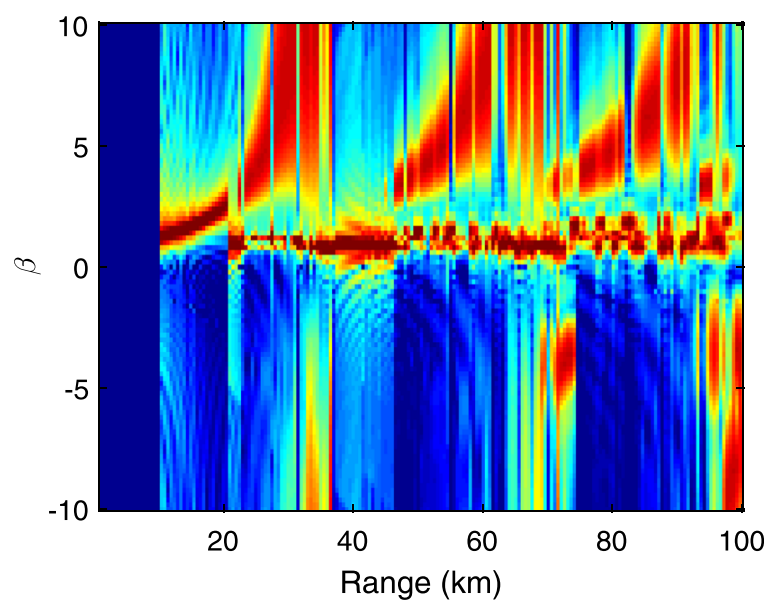

FIG. 5. (Color online) $\hat{E}_{\beta}(r)$ at different source ranges. Other parameters are set at $z_{r}=1000 \mathrm{~m}$ and $z_{s}=500 \mathrm{~m}$. term and thus to correct the approximation used in Eq. (7). This last decomposition will prove to yield a definition of the WI that is an explicit function of range, and will provide a WI prediction consistent with the reference WI distribution.

\section{B. On the phase of the modal pressure}

The eigenmode $\psi_{m}(z, \omega)$ is a real function. It oscillates between the upper and the lower turning points of the mode $m, z_{m}^{+}$and $z_{m}^{-}$. It can thus be decomposed in terms of magnitude and phase. The Wentzel-Kramers-Brillouin (WKB) approximation $^{7,32}$ gives a very good AM-FM decomposition of the eigenmode in terms of a sum of an up-going wave $\Psi_{m}^{-}(z)$ and a down-going wave $\Psi_{m}^{+}(z)$,

$$
\psi_{m}(z, \omega)=\Psi_{m}^{-}(z, \omega)+\Psi_{m}^{+}(z, \omega) .
$$

This approximation is valid within the oscillatory zone and breaks down close to turning points where the vertical wavenumber $k_{z, m}(z, \omega)=\sqrt{\omega^{2} / c(z)^{2}-k_{m}^{2}(\omega)}$ vanishes and the magnitude diverges

$$
\Psi_{m}^{-}(z, \omega)=\frac{C^{-}}{\sqrt{k_{z, m}(z, \omega)}} e^{-i \int_{z_{m}^{-}}^{z} k_{z, m}\left(z^{\prime}, \omega\right) \mathrm{d} z^{\prime}}
$$

and

$$
\Psi_{m}^{+}(z, \omega)=\frac{C^{+}}{\sqrt{k_{z, m}(z, \omega)}} e^{i \int_{z_{m}^{+}}^{z} k_{z, m}\left(z^{\prime}, \omega\right) \mathrm{d} z^{\prime}},
$$

where $C^{ \pm}$are constants. Beyond these turning points, the eigenmode decays exponentially and the wave is evanescent. Inserting Eq. (21) into Eq. (1), the mode $m$ pressure, as a product of two eigenmodes, is then a sum of $2 \times 2=4$ waves indexed by $\mathbf{w}=(\xi, \varepsilon, m)$, with $\varepsilon= \pm 1$ and $\xi= \pm 1$. The phases of those four waves are given by

$$
\begin{aligned}
\phi_{m}^{\xi \varepsilon}\left(z_{r}, z_{s}, r, \omega\right)= & k_{m}(\omega) r+\xi \int_{z_{m}^{\xi}}^{z_{s}} k_{z, m}(z, \omega) \mathrm{d} z \\
& +\varepsilon \int_{z_{m}^{\varepsilon}}^{z_{r}} k_{z, m}(z, \omega) \mathrm{d} z
\end{aligned}
$$

and their magnitudes are smooth positive functions (except around turning points) given by

$$
A_{m}^{\xi \varepsilon}\left(z_{r}, z_{s}, r, \omega\right)=\frac{C^{\varepsilon} C^{\xi}}{\sqrt{k_{z, m}\left(z_{r}, \omega\right) k_{z, m}\left(z_{s}, \omega\right) k_{m}(\omega) r}} .
$$

One notes that, if $(\varepsilon, \xi)=(0,0)$, Eq. (24) is equivalent to Eq. (7). Seabed and water attenuations are ignored here for notation convenience, but could easily be included into the wave magnitude in Eq. (25).

\section{The WI as an explicit function of range}

Using the latter AM-FM decomposition of $\psi_{m}$, the modal pressure appears as a sum of four waves. Then the acoustic intensity is proportional to a sum of $(4 N) ! /(4 N-2) ! 2$ ! interferences between all possible pairs of different waves $\mathbf{w}=(\varepsilon$, $\xi, m)$ and $\mathbf{v}=(\mu, \nu, n)$ with $\mathbf{w} \neq \mathbf{v}$. The WI associated to a pair of waves is then 


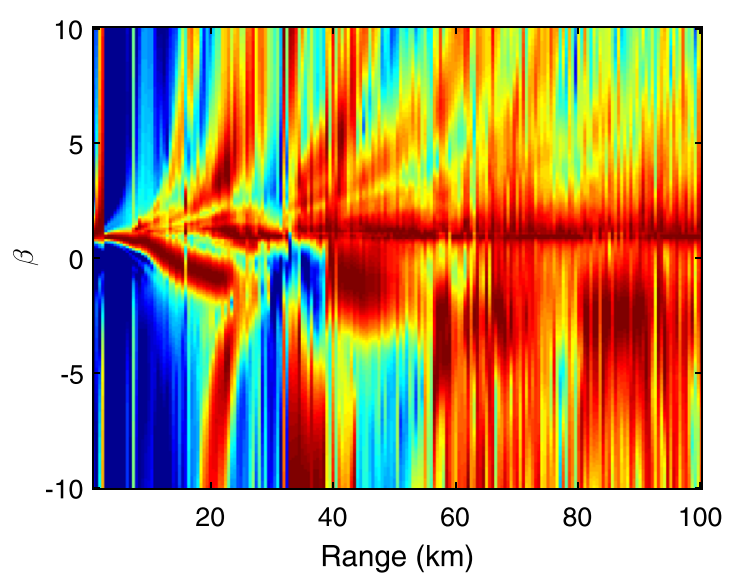

FIG. 6. (Color online) $\hat{E}_{\beta}^{\mathrm{wkb}}(r)$ at different source ranges. Other parameters are set at $z_{r}=1000 \mathrm{~m}$ and $z_{s}=500 \mathrm{~m}$.

$$
\beta_{\mathbf{w v}}\left(z_{s}, z_{r}, r, \omega\right)=-\frac{r}{\omega} \frac{\frac{\delta \Delta \phi_{\mathbf{w v}}\left(z_{s}, z_{r}, r, \omega\right)}{\delta r}}{\frac{\delta \Delta \phi_{\mathbf{w v}}\left(z_{s}, z_{r}, r, \omega\right)}{\delta \omega}},
$$

where $\Delta \phi_{\mathbf{w v}}=\phi_{m}^{\xi \varepsilon}-\phi_{n}^{\mu \nu}$. It is important to note that the waves $\mathbf{w}$ and $\mathbf{v}$ may mutually interfere with $m=n$. We now derive separately the two partial derivatives of Eq. (26).

Starting with the range derivative we find,

$$
\frac{\delta \Delta \phi_{\mathbf{w v}}\left(z_{s}, z_{r}, r, \omega\right)}{\delta r}=\Delta k_{m n}(\omega) .
$$

The result is equivalent to the one resulting from Eq. (6). It does not depend on $\varepsilon$ and $\xi$, but only on mode numbers.

In order to obtain a tractable expression for the partial frequency derivative, we proceed step by step. First, the propagation time $t_{m}^{\xi \varepsilon}$ of the wave $\mathbf{w}$ is given by the stationary phase point $^{33,34}$

$$
\frac{\delta}{\delta \omega}\left[\omega t_{m}^{\xi \varepsilon}-\phi_{m}^{\xi \varepsilon}\right]=0
$$

so that

$$
t_{m}^{\xi \varepsilon}=\frac{\delta \phi_{m}^{\xi \varepsilon}}{\delta \omega} .
$$

Introducing the vertical group slowness $S_{g, m}^{v}(\omega)=\delta k_{z, m}$ $(\omega) / \delta \omega$, and inserting Eq. (24) into Eq. (29),

$$
\begin{aligned}
t_{m}^{\xi \varepsilon}\left(z_{s}, z_{r}, r, \omega\right)= & S_{g, m}^{h}(\omega) r+\xi \int_{z_{m}^{\xi}}^{z_{s}} S_{g, m}^{v}(z, \omega) d z \\
& +\varepsilon \int_{z_{m}^{\varepsilon}}^{z_{r}} S_{g, m}^{v}(z, \omega) d z .
\end{aligned}
$$

Then we can define the effective group slowness, ${ }^{34}$ which is slightly different for each wave,

$$
S_{g, m}^{\xi \varepsilon}\left(z_{s}, z_{r}, r, \omega\right)=\frac{t_{m}^{\xi \xi}\left(z_{s}, z_{r}, r, \omega\right)}{r} .
$$

Inserting Eqs. (27) and (29) into Eq. (26), we identify both the horizontal phase slowness and the effective group slowness, and obtain

$$
\beta_{\mathbf{w v}}\left(z_{s}, z_{r}, r, \omega\right)=-\frac{\Delta S_{p, m n}^{h}(\omega)}{\Delta S_{g, \mathbf{w v}}\left(z_{s}, z_{r}, r, \omega\right)},
$$

with $\Delta S_{g, \mathbf{w v}}=S_{g, m}^{\xi_{\varepsilon}}-S_{g, n}^{\mu \nu}$. The numerator is equivalent to the one in Eq. (10). This means that the four waves $(\varepsilon= \pm 1$, $\xi= \pm 1, m)$ have the same phase velocity which is the horizontal phase velocity of the mode $m$. However, their group slownesses are different, so that Eq. (32) is different from Eq. (10).

The above definition of the WI is intrinsically range and depth dependent. At infinite ranges, the integral terms in Eq. (30) becomes negligible compared to $S_{g, m}^{h} r$. In this case, the four waves with the same mode number almost propagate at the same effective group slowness which is the horizontal group slowness of the mode, so that the usual definition of the travel time $t_{m}=S_{g, m}^{h} r$ is appropriate and the WI as defined in Eq. (10) is a good approximation of the one defined in Eq. (32).

\section{WI distribution in deep water configuration}

Analogous to the modal dominance exposed in Sec. III A, we introduce the waves dominance in the calculation of $E_{\beta}$. Inserting Eq. (21) into Eq. (1), the complex pressure field is a sum of $4 N$ waves with local maxima occurring when adjacent waves interfere constructively. The wave indexed by $\mathbf{w}^{+1}=(\xi, \varepsilon, m+1)$ is defined as adjacent to the wave $\mathbf{w}=(\xi, \varepsilon, m)$. Adjacent waves share the same parameters $\varepsilon$ and $\xi$ and produce constructive interference when they are in phase

$$
\Delta \phi_{\mathbf{w w}^{+1}}\left(z_{r}, z_{s}, r, \omega\right)=2 p \pi .
$$

As shown previously, we track the evolution of $p$ as a function of $m$ and find groups of neighboring waves centered on the equivalent wave $\mathbf{w}_{\mathbf{p}}=\left(\xi_{p}, \varepsilon_{p}, m_{p}+0.5\right)$ that corresponds to integer values of $p$. Each group of neighboring waves behaves almost together like this equivalent wave $\mathbf{w}_{\mathbf{p}}$. As in Sec. III A, the mode number $m_{p}$ is usually not an integer and the physical quantities $\left(k_{m_{p}}, k_{z, m_{p}}, \phi_{m_{p}}^{\varepsilon_{p} \xi_{p}}, A_{m_{p}}^{\varepsilon_{p} \xi_{p}}, \ldots\right)$ associated with the equivalent wave are approximated by interpolation with waves w. This interpolation is a simple onedimensional interpolation over $m$, since $\varepsilon_{p}$ and $\xi_{p}$ always remains \pm 1 .

Then the WI resulting from the interference of two equivalent waves $\mathbf{w}_{\mathbf{p}}$ and $\mathbf{w}_{\mathbf{q}}$ is

$$
\beta_{\mathbf{w}_{\mathbf{p}} \mathbf{w}_{\mathbf{q}}}\left(z_{s}, z_{r}, r, \omega\right)=-\frac{\Delta S_{p, m_{p} m_{q}}^{h}(\omega)}{\Delta S_{g, \mathbf{w}_{\mathbf{p}} \mathbf{w}_{\mathbf{q}}}\left(z_{s}, z_{r}, r, \omega\right)} .
$$

Now the WI depends explicitly on range through the effective group slowness, and implicitly through equivalent waves that denotes wave dominance at a given range.

Putting all together and following the same procedure as in Sec. II B a WI distribution is calculated as the sum of interferences between all equivalent waves. It is referred to as a prediction, noted $\hat{E}_{\beta}^{\mathrm{wkb}}$, and it is plotted in Fig. 6 for the example of Sec. II B. There are two major differences with the prediction $\hat{E}_{\beta}$ in Sec. III A. The modal dominance is not 
equivalent to waves dominance and values of WI given by Eq. (34) can be totally different than those given by Eq. (18), especially for relatively short ranges.

The new $\hat{E}_{\beta}^{\mathrm{wkb}}$ is consistent with the reference $E_{\beta}$. It predicts particular $\beta$-values at the correct ranges. But since the AM-FM decomposition has been performed using the WKB approximation, the waves amplitudes and then the interference excitations can diverge. Even if an empirical threshold has been set to avoid an infinite divergence, amplitude estimations can be an issue. For instance, in Fig. 6, interference at $\beta \approx-1.5$ at range $r=45 \mathrm{~km}$ is overestimated.

\section{SOME PARTICULAR EXAMPLES OF STRIATION PATTERN IN DEEP WATER CONFIGURATION}

The Mediterranean environment given in Table I is still considered. The results obtained here are representative of what could be found in any deep water environment whose SSP has a single minimum. More complex environments with several SSP minimums, such as the North-East Atlantic SSP, would require to recompute the WI distribution, and to be careful with the integral limits in Eq. (24).

The two approximated distributions, $\hat{E}_{\beta}$ and $\hat{E}_{\beta}^{\mathrm{wkb}}$, are now examined at some particular ranges to show interesting features of the deep water striation patterns. Let us remind that $\hat{E}_{\beta}$ uses $(\xi, \varepsilon)=(0,0)$, it corresponds to the usual assumptions, where the frequency dependence of $\psi_{m}$ is ignored, leading to the phase approximation given in Eq. (7). $\hat{E}_{\beta}^{\mathrm{wkb}}$ uses $(\xi, \varepsilon)=( \pm 1, \pm 1)$ and the frequency dependence of $\psi_{m}$ is taken into account, impacting the WI calculation in Eq. (34) and the waves dominance in Eq. (33), but amplitudes can diverge. They are tested against the reference distribution $E_{\beta}$ which is measured using a 2D-FFT on synthetic data generated by running a Parabolic
Equation (PE) using the RAM code. ${ }^{35}$ The environment used for the PE is given in Table I. The final (and non-physical) attenuated bottom layer required to run PE has been set to $4 / 3$ of the water depth.

The source/receiver configurations are chosen to clearly highlight the impact of the group dominance and/or the impact of vertical group slowness on striation patterns. Three cases will be analyzed for a deep source at $z_{s}=500 \mathrm{~m}$ and a shallow source at $z_{s}=10 \mathrm{~m}$. A ray trace for each source depth is provided in Figs. 3(b) and 3(c), respectively, along with the positions of the Horizontal Line Arrays (HLA).

\section{A. Lloyd mirror pattern}

Configuration settings: $z_{s}=500 \mathrm{~m}, \quad z_{r}=1000 \mathrm{~m}$, $r=8.5 \mathrm{~km}, L=1 \mathrm{~km}$ as it is displayed in Fig. 3(b).

For this first case, the HLA is relatively close to the source. At this range, the frequency dependence of the modal eigenfunctions has a huge effect on the phase term in Eq. (24). Figure 7(a) shows a graphic representation of the group dominance using Eqs. (15) and (33). Based on this Fig. 7(a), one finds three equivalent waves $\mathbf{w}_{\mathbf{p}}=\left(\xi_{p}, \varepsilon_{p}, m_{p}\right)$ that verify Eq. (33) whereas no equivalent mode verifies Eq. (15) (the dashed curve does not cross any integer value of $p$ ). Physical properties of those equivalent waves are interpolated as explained in Sec. IIID. Figure 7(b) displays waves and the modes themselves in the group/phase slowness plane. The size of the black markers are proportional to equivalent wave magnitudes. The first observation is that all equivalent waves do not behave like modes, especially the biggest black marker which has a group slowness $0.011 \mathrm{~ms} / \mathrm{m}$ higher than a mode with the same phase slowness. This means that this equivalent wave propagates $25 \mathrm{~m} / \mathrm{s}$ slower than this mode. The vertical (a)

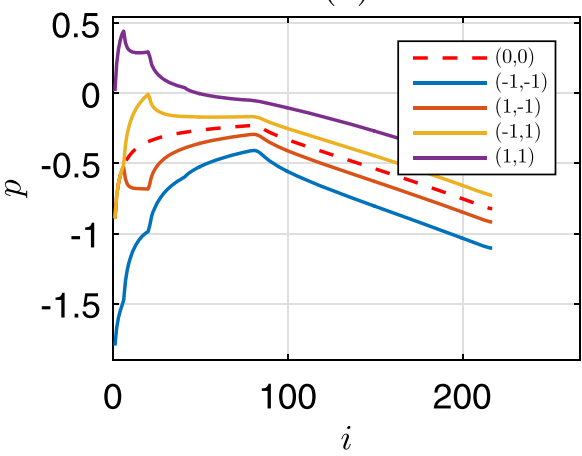

(c)

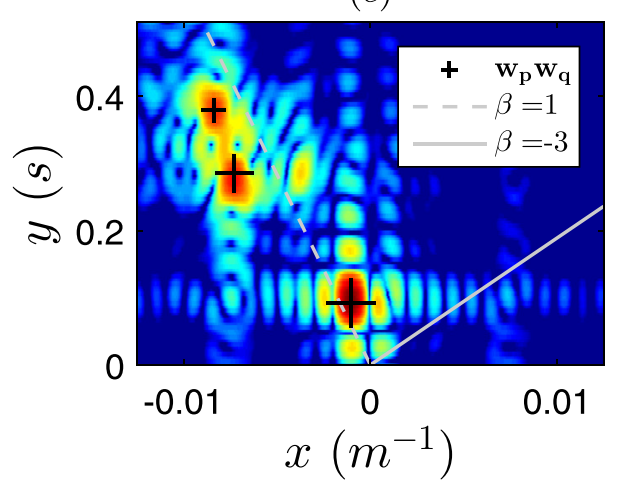

(b)

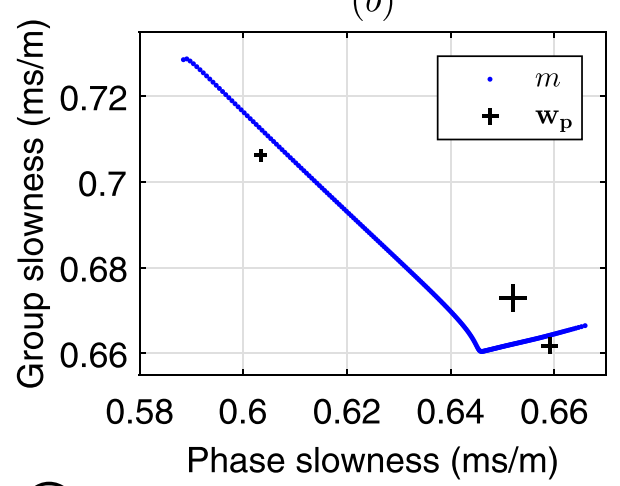

(d)

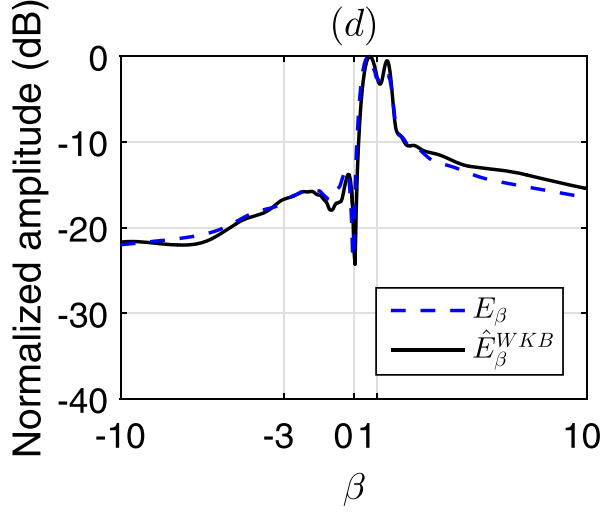

FIG. 7. (Color online) (a) Graphic representation of the group dominance. (b) Modes and equivalent waves at $f=150 \mathrm{~Hz}$ displayed in the phase/ group slowness plane (equivalent modes are not displayed as none have been found). (c) 2D-FFT of $I(r, f)$ with equivalent waves interferences superimposed. (d) The WI distributions $E_{\beta}$ and $\hat{E}_{\beta}^{\mathrm{wkb}}$ ( $\hat{E}_{\beta}$ is not drawn as no equivalent mode has been found). 
distance between black markers and blue dots denotes the contribution of integral terms of Eq. (30). The three equivalent waves produce three interference terms between waves $\mathbf{w}_{\mathbf{p}}$ and $\mathbf{w}_{\mathbf{q}}$. In the background of Fig. 7(c) is the 2D-FFT of the reference range-frequency image $I(r, f)$ computed as in Sec. II B. The locations in the Fourier domain, $\left(x_{\mathbf{w}_{\mathbf{p}} \mathbf{w}_{\mathbf{q}}}, y_{\mathbf{w}_{\mathbf{p}} \mathbf{w}_{\mathbf{q}}}\right)$, of striations resulting from interferences of previous equivalent waves are superimposed as black cross markers. The size of these markers are now proportional to the interference excitations $A_{\mathbf{w}_{\mathbf{p}}} A_{\mathbf{w}_{\mathbf{q}}}$. It coincides with the locations of the observed reference striations. The resulting $\hat{E}_{\beta}^{\mathrm{wkb}}$ is shown in Fig. 7(d) and also matches the reference $E_{\beta}$. Obviously, $\hat{E}_{\beta}$ is not drawn since no equivalent mode has been found.

An interesting property arises here. It is related with the two waves $\mathbf{w}_{\mathbf{p}}=\left(1,1, m_{p}\right)$ and $\mathbf{w}_{\mathbf{q}}=\left(-1,-1, m_{q}\right)$ with $m_{p}$ $\approx 18.5$ and $m_{q} \approx 48.5$. These waves are interfering and building striations controlled by a positive value of $\beta_{\mathbf{w}_{\mathbf{p}} \mathbf{w}_{\mathbf{q}}}$ $\approx 0.6$ while $\beta_{m_{p} m_{q}} \approx-3$. The WI can switch to a positive $\beta$ value from a negative one, just because of the integral terms in Eq. (30). In other words, even if one considers refracted modes which are known to give birth to negative striation slopes, the resulting interference pattern can nevertheless exhibit positive striation slopes. This property is consistent with a Lloyd mirror pattern, where a surface reflected wave interferes with a direct refracted wave. A wave decomposition of the eigenmode gives a fair explanation of this.

\section{B. $\beta$-value close to zero}

Configuration settings: $z_{s}=500 \mathrm{~m}, \quad z_{r}=1000 \mathrm{~m}$, $r=12.5 \mathrm{~km}, L=1 \mathrm{~km}$ as it is displayed in Fig. 3(b).

The HLA is slightly further from the source than in the previous configuration. In this case, as illustrated in Fig. 8(a), one finds two equivalent modes and eight equivalent waves. As shown previously, equivalent waves and equivalent modes are plotted in the group/phase slowness plane in Fig. 8(b). One notes that one equivalent mode and three equivalent waves have their magnitude close to zero: the associated markers in Fig. 8(b) are so small that they cannot be seen. It means that either the source or the receiver is out of the oscillatory zone of the eigenmodes and the waves are evanescent. The two equivalent modes build one interference striation denoted by the red cross marker in Fig. 8(c). It does not coincide with any reference interference striation. The eight equivalent waves give birth to many interference striations that match the striation pattern, in terms of spatial coordinates in the Fourier domain (black cross markers exactly coincide with patch of energy centroids) and of relative interference excitation (relative size of the markers), as illustrated in Fig. 8(c). The two predicted WI distributions $\hat{E}_{\beta}$ and $\hat{E}_{\beta}^{\mathrm{wkb}}$ are shown in Fig. 8(d). Equivalent waves are enough to explain the full complexity of the observed reference striation pattern whereas equivalent modes fail.

This example has been chosen to illustrate a particular case which is related to the two equivalent waves $\mathbf{w}_{\mathbf{p}}$ $=\left(1,1, m_{p}\right)$ and $\mathbf{w}_{\mathbf{q}}=\left(-1,-1, m_{q}\right)$ with $m_{p} \approx 24$ and $m_{q}$ $\approx 24$. The corresponding phase slowness values are $S_{p, m_{p}}^{h}$ $\approx S_{p, m_{q}}^{h} \approx 0.658 \mathrm{~ms} / \mathrm{s}$ and are recognizable in Fig. 8(b). In fact, from ray theory, each equivalent wave is comparable to an eigenray with launching angle $\theta_{s, m_{p}}=\xi_{p} c\left(z_{s}\right) S_{p, m_{p}}^{h}$ and arrival angle $\theta_{r, m_{p}}=\varepsilon_{p} c\left(z_{r}\right) S_{p, m_{p}}^{h}$, where $c(z)$ is the sound speed at depth $z$. Then, the couple $\left(\xi_{p}, \varepsilon_{p}\right)$ may be understood as describing the path of an equivalent wave. For the given configuration the HLA is located at the exact range where rays (or equivalent waves) with launching angles $\theta_{s, m_{p}}$ $=-\theta_{s, m_{q}}$ are eigenrays and energies converge on the HLA (a)

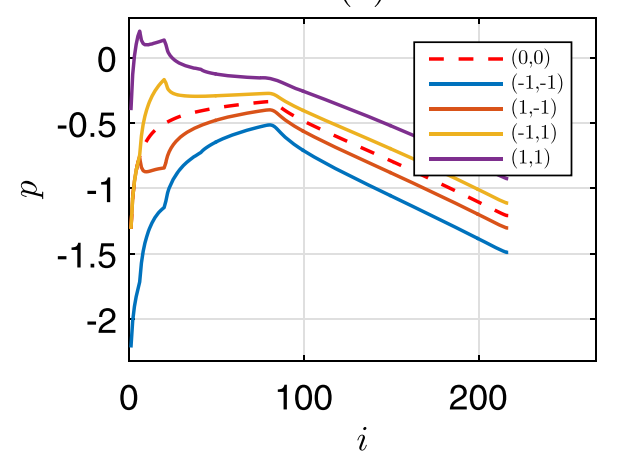

(c)

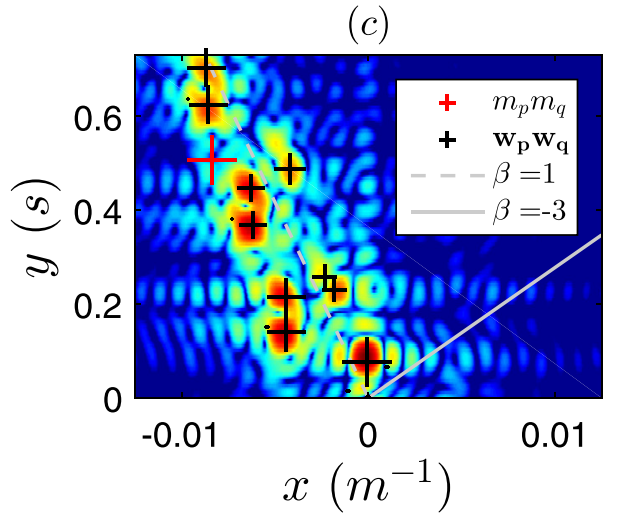

(b)

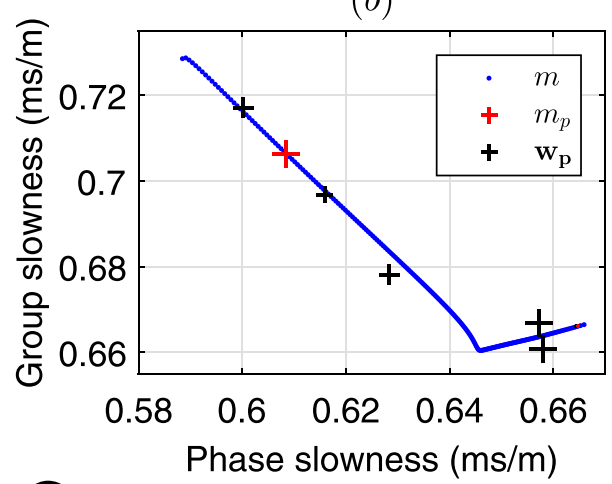

(d)

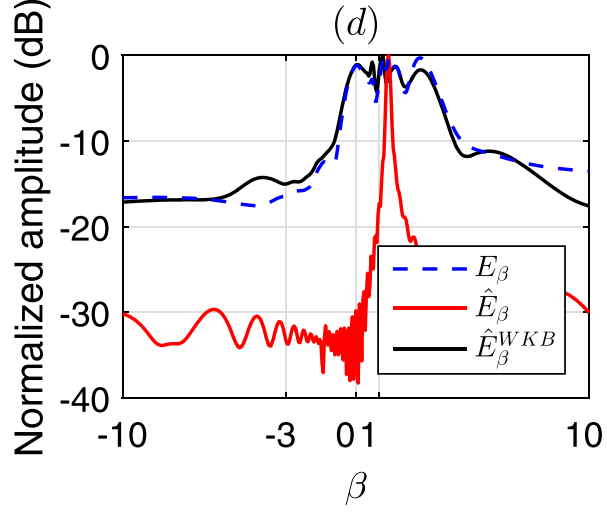

FIG. 8. (Color online) (a) The graphic representation of the group dominance. (b) Modes, equivalent modes and equivalent waves at $f=150 \mathrm{~Hz}$ displayed in the phase/group slownesses plane. (c) 2D-FFT of $I(r, f)$ with equivalent waves/modes interferences superimposed. (d) The WI distributions $E_{\beta}, \hat{E}_{\beta}$, and $\hat{E}_{\beta}^{\mathrm{wkb}}$. 
with different travel times. It is equivalent to say that the two equivalent waves dominate the field with the same phase slowness but with different effective group slownesses. This leads to the $\beta=0$ component in the WI distribution as can be seen in Fig. 8(d), and it is well predicted by $\hat{E}_{\beta}^{\mathrm{wkb}}$.

\section{Convergence zone}

Configuration settings: $z_{s}=10 \mathrm{~m}, \quad z_{r}=500 \mathrm{~m}$, $r=34.5 \mathrm{~km}, L=1 \mathrm{~km}$ as it is displayed in Fig. 3(c).

The source is close to the surface and the HLA is located at $z_{r}=500 \mathrm{~m}$ in the first convergence zone of low order modes (bottom refracted modes). These modes are known to produce interference patterns characterized by a negative $\beta$-value and should constructively interfere on the HLA at some specific ranges (e.g., $r=34.5 \mathrm{~km}$ ). We verify here the pertinence of our method in this specific zone. First of all, the source is close to a pressure release interface, so that the $\xi$-integral term in Eq. (24) is really small. Then waves satisfy $\mathbf{w}=(1, \varepsilon, m) \approx(-1, \varepsilon, m)$. This can be observed in Fig. 9(a) where $(-1,-1)$ and $(1,-1)$ curves or $(-1,1)$ and $(1,1)$ curves overlap. At this range, one observes plenty of equivalent modes/waves and, as expected for a convergence zone, low order equivalent modes/waves may be found. In Fig. 9(b), equivalent waves (black markers) have almost the same slowness as modes (blue dots). Integral terms in Eq. (30) are small compared to $S_{g, m}^{h} r$. However, even if the waves decomposition of the eigenmode does not change much the effective group slowness, it still has a huge impact on wave dominance. Indeed, the equivalent modes (red markers) are completely different from equivalent waves (black markers). Since we are relatively far from the source, the striation pattern is complex and features many overlapping components, as shown in Fig. 9(c). However all these components are well predicted by equivalent wave interferences. The resulting distribution is then well predicted by $\hat{E}_{\beta}^{\mathrm{wkb}}$, whereas $\hat{E}_{\beta}$ still fails to describe the striation pattern, as shown in Fig. 9(d). This demonstrates that equivalent wave interferences accurately predict specific striation patterns arising in the convergence zone. At least for the first convergent zone, equivalent modes do not manage to do so. As a result, the wave decompositions of the eigenmodes can be relevant even at several tens of kilometers. This is because of the groups dominance that more likely explains the observations with equivalent waves than with equivalent modes.

\section{CONCLUSION}

We have considered one of the simplest deep water environments, namely the constant positive barocline velocity gradient profile, topped by a sharp upper thermocline with negative sound-speed gradient. This case, however simple, nicely models the Mediterranean Sea in summer. Even in this elementary case, interferences lead to complex striation patterns that may vary quickly with range or receiver/ source depth. Indeed, the SSP is largely stratified and even at a few Hertz, the modes start to form paths. This involves a WI distribution which is indirectly controlled by the dominance of few groups of waves, and is intrinsically a function of depth and range through the frequency dependence of the eigenmodes. Depending on the configuration, it is essential to take into account these two phenomena until several tens of kilometers. This order of magnitude should be generalizable to most deep water environments since interference cycle (equivalently ray cycle) is about tens of kilometers. (a)

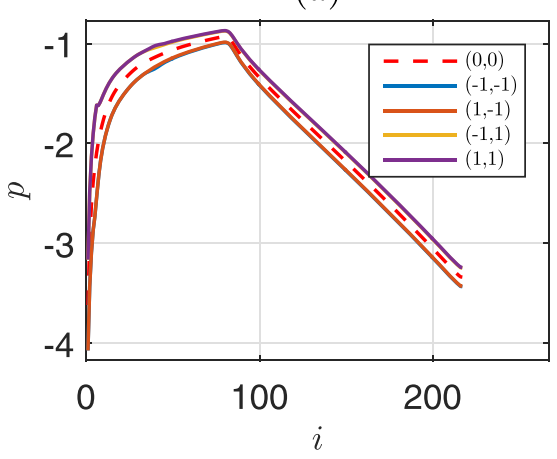

(c)

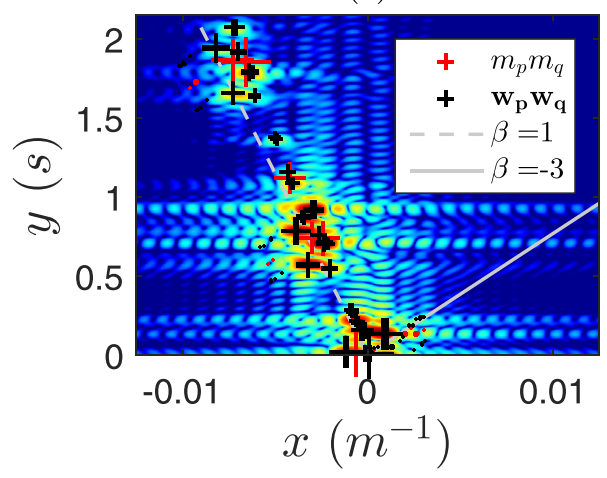

(b)

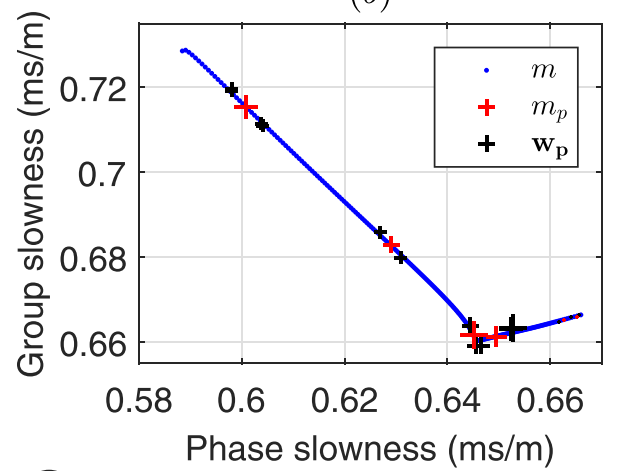

(d)

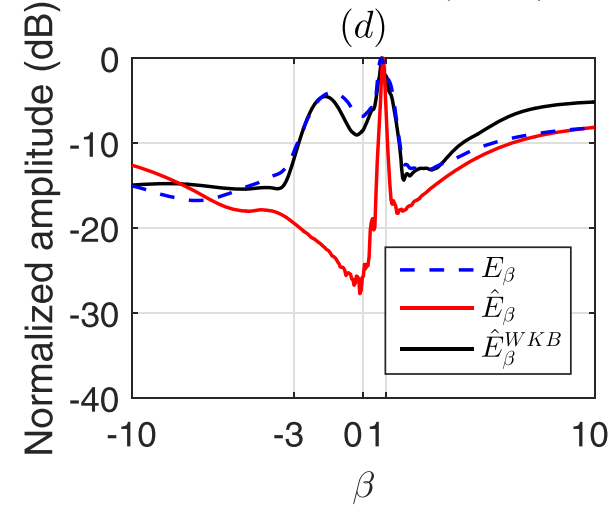

FIG. 9. (Color online) (a) The graphic representation of group dominance. (b) Modes, equivalent modes, and equivalent waves at $f=150 \mathrm{~Hz}$ displayed in the phase/group slownesses plane. (c) 2D-FFT of $I(r, f)$ with equivalent waves/modes interferences superimposed. (d) The WI distributions $E_{\beta}, \hat{E}_{\beta}$, and $\hat{E}_{\beta}^{\mathrm{wkb}}$. 
Then, using the normal mode theory, a WI distribution can be predicted as a function of range and depth. It matches the observed reference distribution from short range interference patterns (Lloyd mirror patterns) to long-range interference patterns along with convergence-shadow zones.

However, for a more complex realistic SSP, the AMFM decomposition of the eigenmodes using the WKB approximation becomes really cumbersome. Moreover, even for a simple SSP like a simple thermocline over a barocline, the interference excitations can be a challenging issue if the empirical threshold avoiding magnitude divergence is mischosen. If the WKB approximation fails or is too complex for complex SSP, signal processing based AM-FM decompositions may be used. A good candidate is the use of Hilbert transform, although this needs to be investigated.

Our work provides a better understanding of the striation patterns in deep water from the normal mode point of view. It allows an accurate prediction of the WI distribution, and could thus be used as the basis of inversion method based on this distribution. We believe it will be particularly useful in the context of source depth discrimination.

\section{ACKNOWLEDGMENTS}

This work was funded by Delegation General de l'Armement and by Thales Underwater Systems. We warmly acknowledge D. Fattaccioli (DGA) for his scientific support. J.B.'s contribution was supported by ENSTA Bretagne (France) and by the Investment in Science Fund at WHOI (USA).

${ }^{1} \mathrm{X}$. Lurton, An Introduction to Underwater Acoustics: Principles and Applications (Springer Science \& Business Media, Berlin, 2002).

${ }^{2}$ D. E. Weston and K. J. Stevens, "Interference of wide band sound in shallow water," J. Sound. Vib. 21(1), 57-64 (1972).

${ }^{3}$ S. D. Chuprov, "Interference structure of a sound field in a layered ocean," in Ocean Acoustics Current State (Nauka, 1982), pp. 71-91.

${ }^{4}$ K. L. Cockrell, "Understanding and utilizing waveguide invariant range frequency striations in ocean acoustic waveguides," Ph.D. thesis, Massachusetts Institute of Technology, Cambridge, 2011.

${ }^{5}$ G. A. Grachev, "Theory of acoustic field invariants in layered waveguides," Acoust. Phys. 39(1), 67-71 (1993).

${ }^{6} \mathrm{C}$. H. Harrison, "The relation between the waveguide invariant, multipath impulse response, and ray cycles," J. Acoust. Soc. Am. 129(5), 2863-2877 (2011).

${ }^{7}$ L. Brekhovskikh and Y. Lysanov, Fundamentals of Ocean Acoustics (Springer Science \& Business Media, Berlin, 2013).

${ }^{8}$ F. B. Jensen, W. A. Kuperman, M. B. Porter, and H. Schmidt, Computational Ocean Acoustics (Springer Science \& Business Media, Berlin, 2011).

${ }^{9}$ A. M. Thode, "Source ranging with minimal environmental information using a virtual receiver and waveguide invariant theory," J. Acoust. Soc. Am. 108(4), 1582-1594 (2000).

${ }^{10}$ K. L. Cockrell and H. Schmidt, "Robust passive range estimation using the waveguide invariant," J. Acoust. Soc. Am. 127(5), 2780-2789 (2010).

${ }^{11}$ K. D. Heaney, "Rapid geoacoustic characterization using a surface ship of opportunity," IEEE J. Ocean. Eng. 29(1), 88-99 (2004).
${ }^{12}$ J. E. Quijano, L. M. Zurk, and D. Rouseff, "Demonstration of the invariance principle for active sonar," J. Acoust. Soc. Am. 123(3), 1329-1337 (2008).

${ }^{13}$ L. M. Zurk and D. Rouseff, "Striation-based beamforming for active sonar with a horizontal line array," J. Acoust. Soc. Am. 132(4), EL264-EL270 (2012).

${ }^{14}$ Y. Le Gall and J. Bonnel, "Separation of moving ship striation patterns using physics-based filtering," Traitement du Signal 30(3-5), 149-168 (2013) [in French].

${ }^{15}$ D. Zhi Gao, N. Wang, and H. Zhong Wang, "A dedispersion transform for sound propagation in shallow water waveguide," J. Comput. Acoust. 18(03), 245-257 (2010).

${ }^{16}$ J. Bonnel, G. Le Touze, B. Nicolas, and J. I. Mars, "Physics-based timefrequency representations for underwater acoustics: Power class utilization with waveguide invariant approximation," IEEE Signal Process. Mag. 30(6), 120-129 (2013).

${ }^{17}$ A. B. Baggeroer, "Estimation of the distribution of the interference invariant with seismic streamers," AIP Conf. Proc. 621, 151-170 (2002).

${ }^{18}$ D. Rouseff and R. C. Spindel, "Modeling the waveguide invariant as a distribution," AIP Conf. Proc. 621, 137-150 (2002).

${ }^{19}$ T. C. Yang, "Beam intensity striations and applications," J. Acoust. Soc. Am. 113(3), 1342-1352 (2003).

${ }^{20}$ A. Turgut and L. T. Fialkowski, "Depth discrimination using waveguide invariance," J. Acoust. Soc. Am. 132(3), 2054-2054 (2012).

${ }^{21}$ Julien Bonnel, "Analysis of low frequency $(0-150 \mathrm{~Hz})$ modal dispersion for oceanic environment characterization," Ph.D. thesis, Institut National Polytechnique de Grenoble - INPG (2010) [in French].

${ }^{22}$ C. Cho, H.-C. Song, and A. Thode, "Extension of the array invariant to deep-water environments," J. Acoust. Soc. Am. 141(5), 4049-4049 (2017).

${ }^{23}$ R. Emmetiere, J. Bonnel, M. Gehant Pernot, and T. Chonavel, "Source depth discrimination using the array invariant," J. Acoust. Soc. Am. 141(5), 3989-3989 (2017).

${ }^{24}$ L. Ian-Qian, L. Zheng-Lin, and Z. Ren-He, "Applications of waveguide invariant theory to the analysis of interference phenomena in deep water," Chinese Phys. Lett. 28(3), 034303 (2011).

${ }^{25}$ M. B. Porter, "The kraken normal mode program," Technical report, Naval Research Lab, Washington DC, 1992.

${ }^{26}$ Y. Le Gall and J. Bonnel, "Separation of moving ship striation patterns using physics-based filtering," Proc. Meet. Acoust. 19(1), 070073 (2013).

${ }^{27}$ J. Du, Y. Zheng, Z. Wang, H. Cui, and Z. Liu, "Passive acoustic source depth discrimination with two hydrophones in shallow water," in OCEANS 2016, Shanghai (April 2016), pp. 1-6.

${ }^{28} \mathrm{~K}$. M. Guthrie and C. T. Tindle, "Ray effects in the normal mode approach to underwater acoustics," J. Sound Vib. 47(3), 403-413 (1976).

${ }^{29} \mathrm{C}$. T. Tindle and K. M. Guthrie, "Rays as interfering modes in underwater acoustics," J. Sound Vib. 34(2), 291-295 (1974).

${ }^{30}$ F. R. DiNapoli and R. L. Deavenport, "Numerical models of underwater acoustic propagation," in Ocean Acoustics (Springer, New York, 1979), pp. 79-157.

${ }^{31}$ K. M. Guthrie, "The connection between normal modes and rays in underwater acoustics," J. Sound Vib. 32(2), 289-293 (1974).

${ }^{32} \mathrm{C}$. L. Bartberger, "The computation of complex normal mode eigenvalues in underwater acoustic propagation," in Computational Acoustics: Algorithms and Applications, edited by D. Lee, R. Sternberg, and M. Schultz (Elsevier Science Publishers, North-Holland, 1988), pp. 149-159.

${ }^{33}$ C. Clay and H. Medwin, Acoustical Oceanography: Principles and Applications (Wiley, New York, 1977).

${ }^{34} \mathrm{P}$. Pignot, "Spatio-temporal characterization of the underwater acoustic propagation: Group and phase velocities reconstruction," Traitement Signal 14(3), 255-274 (1997).

${ }^{35}$ M. D. Collins, "User's guide for ram versions 1.0 and $1.0 \mathrm{p}$," Naval Research Lab, Washington, DC (1995). 